\title{
AVALIAÇÃO DO ESTADO HIDROELETROLÍTICO DE CRIANÇAS PRATICANTES DE EXERCÍCIO FÍSICO E RECOMENDAÇÃO DE HIDRATAÇÃO
}

\author{
DRA. CLAUDIA ALTMAYER PERRONE \\ Doutora em Ciências do Movimento Humano pela Escola de Educação Física \\ da Universidade Federal do Rio Grande do Sul (ESEF/NFRGS) \\ (Porto Alegre - Rio Grande do Sul - Brasil) \\ e-mail: cperrone@terra.com.br \\ DRA. FLAVIA MEYER \\ Professora Associada da Escola de Educação Física (ESEF) da Universidade Federal \\ do Rio Grande do Sul (UFRGS), Doutorado na McMaster University (Canadá) \\ Laboratório de Pesquisa do Exercício (LAPEX/ESEF/UFRGS) \\ (Porto Alegre - Rio Grande do Sul - Brasil) \\ e-mail: flaviameyer@uol.com.br
}

\begin{abstract}
RESUMO
Este estudo aborda as características termorregulatórias, recursos de prevenção e de diagnóstico do déficit hidroeletrolítico e danos causados pela prática do exercício no calor em crianças. Exercitar-se no calor pode levar a um aumento da temperatura central, e comprometer o desempenho e a saúde das crianças. O sistema termorregulatório delas está em desenvolvimento; e a eliminação do calor, por evaporação do suor, fica prejudicada, já que as crianças apresentam menores taxa de sudorese e concentração de eletrólitos no suor do que os adultos. A avaliação do estado de hidratação pré-exercício pode ser importante para prevenir que a criança inicie o exercício desidratada. Além disso, considerando que muitas vezes elas não bebem a quantidade de líquido necessária pra evitar a desidratação durante o exercício, as crianças devem ser educadas a se hidratar antes, durante e após o exercício, especialmente no calor.
\end{abstract}

PALAVRAS-CHAVE: Termorregulação; desidratação; suor; eletrólitos. 


\section{INTRODUÇÃO}

As altas temperaturas e umidade relativa do ar podem ser prejudiciais tanto à saúde quanto ao desempenho na prática de exercício, principalmente daquelas crianças que ficam expostas ao estresse térmico nas regiões tropicais ou durante os meses de verão nos países de clima temperado, e que participam de atividade física e esportes competitivos por períodos prolongados (BERGERON; WALLER; MARINIK, 2006; RIVERA-BROWN et al., 2006).

A termorregulação é considerada o conjunto dos sistemas responsáveis pela regulação da temperatura corporal, e tem como função o equilíbrio entre a produção (termogênese) e a dissipação (termodispersão) do calor central, para manter a temperatura corporal interna em aproximadamente $36,5^{\circ} \mathrm{C}$. A principal via de resfriamento do organismo durante o exercício no calor é a sudorese. A eficiência da evaporação do suor depende umidade relativa do ar, da velocidade do vento, de maneira que, quando a umidade relativa do ar está alta prejudica a evaporação do suor e consequentemente o resfriamento corporal.

A determinação do volume e da composição do suor perdido é importante para posterior estimativa das necessidades de reposição de fluidos e eletrólitos de indivíduos que praticam atividades físicas prolongadas ou intensas e intermitentes. A maioria dos estudos que avaliam as perdas de água e eletrólitos durante o exercício é desenvolvida com adultos (MAUGHAN et al., 2004, 2005; PALACIOS; WIGERTZ; WEAVER, 2003; PATTERSON; GALLOWAY; NIMMO, 2000; SHIRREFFS et al., 2005; VERDE et al., 1982); e alguns poucos, com crianças. Nos estudos que investigam crianças, os protocolos envolvem situações em laboratório (MEYER et al., I 992; MEYER; BAR-OR; WILK, I995; MEYER et al., 2007) ou ao ar livre (BERGERON;WALLER; MARINIK, 2006; BERGERON et al., 2009, RIVERA-BROWN et al., I999, 2006, 2008; RODRÍGUEZ SANTANA et al., I995; WILK; RIVERA-BROWN; RODRIGUEZ SANTANA, 2007); e as condições e sessões de exercício são padronizadas. Em laboratório, a temperatura ambiente e a umidade do ar são estabelecidas e controladas através de câmara ambiental. Nessas situações, as crianças exercitaram-se; e foram avaliados o grau de desidratação, a taxa de sudorese, o balanço hídrico e as respostas termorregulatórias. Nos estudos realizados em laboratórios, também foi avaliada a perda de eletrólitos no suor.

O maior volume de sudorese em relação à ingestão de líquidos durante o exercício causa a hipohidratação. Por isso, o cuidado com a hidratação durante o exercício físico e o esporte tem sido apontado como uma maneira de garantir um bom desempenho e evitar problemas com a saúde. $\bigcirc$ equilíbrio hidroeletrolítico depende de fatores como a composição e a quantidade de suor durante o exercício; 
a composição e a quantidade de líquido ingerido; a duração e o tipo de exercício; a palatabilidade das bebidas; além de características individuais como a maturação e a aclimatação (MEYER; BAR-OR, 1994).

Esta revisão aborda as respostas termorregulatórias ao exercício no calor, bem como métodos para prevenção e identificação de hipohidratação, déficit hidroeletrolítico e dos danos causados pela prática do exercício físico no calor em crianças. Para a elaboração desta revisão, foram selecionados artigos publicados entre 1990 e 20 I 0 no banco de dados MEDLINE que abordassem termorregulação, equilíbrio hidro-eletrolítico durante a prática de exercício físico em crianças. As palavras-chave utilizadas foram: dehydration, thermoregulation, electrolyte loss, exercise, children.

\section{TERMORREGULAÇÃO, SUDORESE E BALANÇO HIDROELETROLÍTICO NA CRIANÇA}

O calor absorvido do meio ambiente pelo organismo e o calor produzido pela contração muscular precisam ser eliminados do organismo para a manutenção da temperatura interna. $O$ calor interno do organismo, que reflete a temperatura central, é transferido para a periferia (pele) através do fluxo sanguíneo. Na pele, o calor pode ser dissipado por condução, convecção, radiação, bem como por evaporação do suor (FALK, 1998; FALK; DOTAN, 2008; INOUE; KUWAHARA; ARAKI, 2004; ROWLAND, 2008). Quando a temperatura ambiente é superior à temperatura da pele, a perda de calor por radiação e convecção ficam prejudicadas, devendo ocorrer principalmente a evaporação do suor (KOVACS, 2006).

As crianças apresentam diferenças na magnitude de suas respostas termorregulátorias em relação aos adultos. Elas conseguem eliminar o calor por convecção de forma satisfatória; no entanto, podem transpirar até 2,5 vezes menos do que os adultos (MEYER et al., 2007).

O sistema termorregulatório de pré-púberes é distinto e está em desenvolvimento quando comparado com o de púberes e de adultos. As características físicas (estatura, massa corporal, adiposidade, relação superfície/massa corporal, glândulas sudoríparas), maturacionais, fisiológicas (metabolismo, circulação e mecanismo de suor) e hormonais de um indivíduo podem influenciar a capacidade do organismo de dissipar e proteger-se do calor (BAR-OR; ROWLAND, 2004; INBAR et al., 2004).

A sudorese não está totalmente desenvolvida nos meninos pré-púberes, possivelmente, devido a ausência de maturação em função da falta de secreção dos hormônios masculinos, além disso, as habilidades das glândulas sudoríparas não se desenvolvem de forma uniforme em todo o corpo durante o crescimento e a maturação (INOUE et al., 2004). Segundo Falk, Bar-Or e MacDougall (1992), o 
tamanho da glândula sudorípara parece estar relacionado com a idade e a altura do indivíduo; e, durante o crescimento e a maturação deste, o aumento do tamanho da glândula está associado ao aumento dos estímulos colinérgico e adrenérgico. Outros fatores que também podem colocar a criança em desvantagem termorregulatória no exercício praticado no calor são: maior produção de calor gerada pelo maior custo metabólico de locomoção, e menor débito cardíaco e volume sanguíneo o que prejudica a eliminação de calor interno pela pele através da convecção (FALK, 1998).

A taxa de sudorese, mesmo nas crianças, é influenciada pelo estresse térmico do ambiente, duração do exercício, além da variabilidade individual. Dois estudos (RIVERA-BROWN et al., 1999; RODRÍGUEZ SANTANA et al., 1995) com protocolos de exercícios intermitentes ao ar livre no calor com alto índice de estresse térmico (WBGT) $\left(30^{\circ} \mathrm{C}\right.$ WBGT) e com duração de $1,2 \mathrm{~h}$ encontraram taxa de sudorese entre 0,5-0,7 I/h. Em protocolos mais curtos em câmara ambiental (40$42^{\circ} \mathrm{C}$ ), (INBAR et al., 2004; MEYER et al., 1992), a taxa de sudorese descrita foi inferior a $0,5 \mathrm{l} / \mathrm{h}$.

A taxa de sudorese, em qualquer condição ambiental ou intensidade de exercício, é significativamente menor em meninos pré-púberes do que em póspúberes, quando corrigida pela superfície de massa corporal. No entanto, quando expressa por quilograma de massa corporal, é similar entre os grupos maturacionais (FALK; BAR-OR; MACDOUGALL, 1992). Em contraste, parece não haver diferenças na taxa de sudorese entre meninas e mulheres, durante o exercício em ambiente quente e úmido (DRINKWATER et al., 1977; RIVERA-BROWN et al., 2006). Ademais, parece não haver diferenças entre os gêneros na taxa de sudorese quando as crianças apresentam similares graus de atividade física e não são aclimatadas (MEYER et al., 1992).

Meninos pré-púberes, quando comparados com adultos, apresentam menor produção de suor relativa ao aumento da temperatura retal; menor taxa de sudorese por glândulas; maior perda de calor evaporado pelo suor quando normalizado pela massa corporal; e maior eficiência da sudorese (perda por evaporação/sudorese total) durante exercício no calor (INBAR et al., 2004). Assim como os púberes, eles também apresentam um menor acúmulo de calor quando comparados com pós-púberes (FALK; BAR-OR; MACDOUGALL, 1992). Possivelmente isso ocorra porque as crianças produzem gotas de suor menores e menos espaçadas, resultando em um maior resfriamento; e porque as gotas de 
suor dos adultos, por serem maiores, podem escorrer pela pele e não resfriar tão eficientemente (INBAR et al., 2004).

A análise da composição do suor de crianças que praticam atividade física no calor pode prever o risco de perdas de eletrólitos e ajudar na prescrição de reposição. O sódio é o cátion mais abundante do espaço extracelular e a sua concentração plasmática exerce um papel fundamental no equilíbrio hídrico, ele é o principal eletrólito perdido pelo plasma através do suor. $O$ cloreto é o ânion mais abundante também presente principalmente no meio extracelular. A avaliação dos eletrólitos no suor pode ser necessária para avaliar riscos de desequilíbrios eletrolíticos provocados por atividades que causam sudorese intensa. Temos desenvolvido pesquisas com jovens praticando diferentes modalidades esportivas no calor e temos alguns resultados preliminares. Os estudos encontrados na literatura durante o exercício em cicloergômetro no calor, dentro de uma câmara ambiental, indicam que crianças pré-púberes, independentemente do gênero, apresentam uma menor concentração de $\mathrm{Na}^{+}, \mathrm{Cl}^{-}$e $\mathrm{K}^{+}$quando comparadas com os púberes (MEYER et al., 1992). Ver Tabela I.

A concentração de lactato no suor também depende do estado maturacional das crianças e da duração do exercício. Tal concentração é maior nas crianças do que nos adultos, não havendo diferenças entre pré-púberes e púberes (FALK et al., |99|, MEYER et al., 2007). Já a concentração de amônia não sofre diferença após duas sessões de 20 min de exercício (MEYER et al., 2007). Ver Tabela I.

TABELA I . Concentração de eletrólitos, lactato e amônia

(em mEq·L-1) no suor de pré-púberes e púberes.

\begin{tabular}{llllll}
\hline & $\mathrm{Na}^{+}$ & $\mathrm{Cl}^{-}$ & $\mathrm{K}^{+}$ & Lactato & Amônia \\
\hline Pré-púberes & $25-35$ & $15-20$ & 12 & \multirow{2}{*}{$14-24$} & \multirow{2}{*}{$3,9-4,7$} \\
\cline { 1 - 4 } Púberes & $35-40$ & $35-40$ & $10-15$ & & \\
\hline
\end{tabular}

Adaptada de MEYER et al., 1992 e 2007.

\section{AVALIAÇÃO DO ESTADO DE HIDRATAÇÃO}

A avaliação do estado de hidratação pré-treinamento ou competição é importante, principalmente para os jovens atletas que participam de modalidades esportivas em que a categoria de competição é determinada pelo peso corporal, já que estão propensos a iniciar a atividade desidratados. $\bigcirc$ diagnóstico do estado de hidratação pode ser feito mediante a avaliação clínica ou de alterações na massa corporal e nos marcadores sanguíneos e urinários. 
A avaliação clínica por meio do reconhecimento de sinais e sintomas é outra forma de avaliar a desidratação durante o exercício no calor. Os primeiros sintomas de hipohidratação são sede e desconforto com o calor. Na hipohidratação leve a moderada, um indivíduo adulto pode apresentar como sinais e sintomas cansaço, cãibra, apatia e pele vermelha. Com um maior déficit de água, pode apresentar tontura, dor de cabeça, vômito, náuseas, sensação de calor na cabeça e no pescoço, tremores, redução de desempenho, dispneia, perda de apetite, sede, intolerância ao calor, e urina mais escura e em menor quantidade. Já com hipohidratação severa, o indivíduo pode apresentar dificuldade de engolir, perda de equilíbrio, pele seca e murcha, olhos afundados e visão fosca, dor para urinar, pele dormente, delírio e espasmos musculares (BAR-OR; ROWLAND, 2004; CASA et al., 2000).

Medidas de massa corporal podem ser uma forma sensível de determinar o estado de hidratação após o exercício, se os cuidados para sua aferição forem tomados antes e depois do exercício, tais como urinar e evacuar antes, usar o mínimo de roupa possível e nenhum calçado, e estar com o corpo seco (AMERICAN COLLEGE OF SPORTS MEDICINE, 2007). A hipohidratação induzida pelo exercício pode ser classificada de acordo com o percentual de perda de peso em leve $(<4 \%)$, moderada (5-8\%) e severa (8- I0\%) (MEYER, 1993).

A determinação do estado de hidratação por parâmetros sanguíneos, como a osmolaridade, é sensível e mais utilizada em pesquisa (QUINTON; TORMEY, 1976; SAWKA, 1992). Num estudo (POPOWSKI et al., 200 I) com atletas adultos, a desidratação de $1 \%$ em 40 min de exercício foi suficiente para aumentar 7 $\mathrm{mOsm} \cdot \mathrm{L}^{-1}$ na osmolaridade sanguínea.

Os marcadores urinários mais utilizados para a verificação do estado de hidratação são osmolaridade, gravidade específica da urina (GEU) e coloração (AMERICAN COLLEGE OF SPORTS MEDICINE, 2007; ARMSTRONG et al., I994, 1998, 2005; CASA et al., 2000; OPPLINGER et al., 2005; SHIRREFFS, 2003).

A Tabela 2 apresenta a classificação do estado de hidratação baseado na alteração de massa corporal e de marcadores urinários. Não existe na literatura valores de referência específico par crianças para estes indicadores, sendo assim, sugerimos o uso dos valores de adultos para classificar o grau de hidratação na população pediátrica. 
TABELA 2. Parâmetros para determinação do estado de hidratação de acordo com as alterações de massa corporal e com parâmetros urinários.

\begin{tabular}{|c|c|c|c|c|}
\hline Parâmetro & $\begin{array}{l}\text { \% Alteração de } \\
\text { massa corporal }\end{array}$ & $\begin{array}{l}\text { Osmolaridade de } \\
\text { urina }\left(\mathrm{mOsm} \cdot \mathrm{kg}^{-1}\right)\end{array}$ & $\begin{array}{l}\text { Gravidade espe- } \\
\text { cífica da urina }\end{array}$ & $\begin{array}{l}\text { Coloração da } \\
\text { urina }\end{array}$ \\
\hline Bem hidratado & $+1--1$ & $<400$ & $<1.010$ & $1-2$ \\
\hline $\begin{array}{l}\text { Hipohidratação } \\
\text { leve }\end{array}$ & $-1--3$ & $400-700$ & $1.010-1.020$ & $3-4$ \\
\hline $\begin{array}{l}\text { Hipohidratação } \\
\text { moderada }\end{array}$ & $-4--5$ & $700-1050$ & $1.020-1.030$ & $5-6$ \\
\hline $\begin{array}{l}\text { Hipohidratação } \\
\text { severa }\end{array}$ & $<-5$ & $>1050$ & $>1.030$ & $>6$ \\
\hline
\end{tabular}

Adaptada de Armstrong et al., 1998 e Casa et al., 2000.

Utilizando parâmetros urinários (osmolaridade, condutividade, coloração e GEU), Kutlu e Guler (2006) avaliaram meninos atletas de taekwon-do e concluíram que, durante os I I dias de concentração pré-competição, eles estavam desidratados pela manhã. Stover e colaboradores (2006) também utilizaram parâmetros urinários para avaliar o estado de hidratação de jovens atletas de futebol americano. Durante uma semana, a gravidade específica da urina foi medida antes do treinamento da manhã; e, na semana seguinte, os atletas foram orientados sobre hidratação e receberam $1200 \mathrm{ml}$ de água ou bebida esportiva para serem consumidos à noite e pela manhã. Observou-se que, durante a semana em que não houve intervenção, pelo menos $60 \%$ dos atletas estavam desidratados, com a gravidade específica da urina acima de 1.020; e, após a orientação de hidratação, houve uma melhora significativa do seu estado de hidratação, com a redução da gravidade específica da urina de 1.021 para $1.016(p<0,01)$.

\section{EQUILÍBRIO HÍDRICO}

As crianças, mesmo quando thes é oferecida água ad libitum durante o exercício, não bebem uma quantidade suficiente para repor suas perdas pelo suor, causando, assim, hipohidratação (BAR-OR et al., 1980; RODRÍGUEZ SANTANA et 
al., 1995; WILK; BAR-OR, 1996). Mínimos graus de desidratação já são suficientes para afetar o tempo de tolerância à prática de exercício no calor; e a redução de $1 \%$ do peso corporal em crianças é suficiente para reduzir o volume sanguíneo e a capacidade aeróbica, e aumentar a temperatura central (AMERICAN ACADEMY OF PEDIATRICS, 2005; WILK et al., 2002). A perda de 2\% do peso corporal sem o consumo de líquidos pode afetar habilidades específicas do basquete em jovens atletas (DOUGHERTY et al., 2006).

Muitas vezes, as práticas de hidratação adotadas por atletas jovens são inadequadas para manter seu estado de hidratação durante um treinamento ou uma competição. Nichols et al. (2005) sugerem que os atletas jovens, em momentos de treino ou competição, não aplicam os conhecimentos que adquiriram sobre práticas de hidratação.

A ingesta voluntária de água não é suficiente para manter o balanço hídrico nem o estado de euhidratação, mesmo de crianças cronicamente aclimatadas e que praticam exercício no calor, ao ar livre, na sombra ou no sol (RODRÍGUEZ SANTANA et al., 1995); ou de jovens triatletas em provas simuladas de duatlon (IULIANO et al., 1998).

Quando são adicionados sabor, carboidrato $(\mathrm{CHO})$ e eletrólitos à bebida, ocorre um aumento de 30 a 45\% no seu consumo comparado com o da água, reduzindo a desidratação voluntária (WILK; BAR-OR, 1996) no entanto, não há alteração nas respostas termorregulatórias (RIVERA-BROWN et al., 1999). Além disso, o consumo de bebidas esportivas por crianças durante o exercício no calor não altera a intensidade da sede nem causa desconforto estomacal (MEYER; BAR-OR; WILK, 1995).

A reposição de líquidos durante o exercício ao ar livre no calor e suas respostas termorregulatórias foram estudadas em jovens tenistas durante 120 min de treinamento em quadra (BERGERON; WALLER; MARINIK, 2006). Nesse estudo, quando consumiram bebidas com $\mathrm{CHO}$ e eletrólitos, os atletas mantiveram a temperatura central significativamente mais baixa do que com o consumo de água. Num estudo subsequente (BERGERON et al., 2009), não foram encontradas diferenças na temperatura central, na taxa de estresse térmico e no índice de dano fisiológico (relação temperatura central e frequencia cardíaca) de jovens atletas após duas sessões seguidas de 80 min de exercício no calor $\left(33^{\circ} \mathrm{C}\right.$ e $48 \%$ UR), com um intervalo de I h entre elas. Os autores sugerem que o consumo de bebida com $\mathrm{CHO}$ e eletrólitos em um volume suficiente para mantê-los euhidratados durante o intervalo foi o responsável pelo retorno da temperatura central a valores basais (pré-sessão I de exercício). 


\section{RECOMENDAÇÃO DE HIDRATAÇÃO PARA CRIANÇAS}

Até o presente momento, não existe um consenso sobre recomendação de hidratação para crianças e adolescentes que praticam atividade física no calor. Estima-se que o balanço hídrico de crianças sedentárias seja de I,6 I por dia (PETRIE; STOVER; HORSWILL, 2004). Sabe-se que, durante as sessões de treinamento ou competições, as crianças e os adolescentes sofrem um aumento das perdas hidroeletrolíticas pelo suor, aumentando a necessidade de consumo de líquido.

Com o objetivo de iniciar um treinamento ou uma competição e hidratado, cada atleta, mesmo sendo criança ou adolescente, deve desenvolver e treinar uma rotina de hidratação. Recomenda-se iniciar o processo de hidratação $4 \mathrm{~h}$ antes do exercício, consumindo aproximadamente $5-7 \mathrm{ml} / \mathrm{kg}$ de peso corporal (AMERICAN DIETETIC ASSOCIATION, DIETITIANS OF CANADA, AMERICAN COLLEGE OF SPORTS MEDICINE, 2009).

As bebidas oferecidas aos atletas devem estar acessíveis, em garrafas adequadas e de fácil alcance, e em baixa temperatura. Durante o exercício, recomenda-se que a ingesta de líquidos ocorra de forma sistemática e que o volume ingerido seja de acordo com a taxa de sudorese. A água é uma boa opção quando a duração do exercício for inferior a 60 min e as condições ambientais estiverem adequadas à prática do exercício.

Quando a duração do exercício ultrapassar 90 min e a intensidade do exercício for de moderada a alta (verificada pela taxa de percepção ao esforço ou pela frequência cardíaca), recomenda-se adicionar $\mathrm{CHO}$ (6-8\%), eletrólitos (sódio 20-25 $\mathrm{mEq} / \mathrm{L})$ e sabor à bebida para promover uma maior absorção de água e aumentar a palatabilidade (MEYER; BAR-OR; WILK, 1995).

Após o exercício, água e sódio devem ser repostos caso haja perda significativa dos mesmos (SHIRREFFS, 200 I). Segundo o American College of Sports Medicine (2007), 1,5\% do peso perdido deve ser reposto. Recomenda-se também o consumo de lanches para aumentar a sede e ajudar na retenção de sódio e líquido pelo organismo.

\section{CONCLUSÃO}

A prática de exercício físico deve ser sempre incentivada à criança; no entanto, alguns cuidados devem ser tomados para que essa prática seja segura quando no calor. As crianças, embora apresentem uma taxa de sudorese e uma perda de eletrólitos pelo suor menores que as dos adultos, estão propensas à desidratação e aos seus possíveis danos tanto ao desempenho quanto a saúde. A avaliação do estado 
de hidratação pré-exercício pode ser necessária, já que os jovens não repõem o que perdem durante o exercício e já iniciam a próxima sessão desidratados. Sendo assim, é importante educá-las para o consumo de líquidos antes, quando necessário durante, e após o exercício no calor.

\section{Assessment of hydroelectrolytic status and hydration recommendations for children during exercise}

ABSTRACT: This study addresses thermoregulatory characteristics and provides resources for prevention and diagnosis of hydroelectrolyte deficits and damage resulting from exercise in the heat by children. Exercise in the heat can lead to an increase in core temperature, thus impairing children's performance and health. The thermoregulatory system is still developing in childhood, and the elimination of the heat produced during physical exercise by sweat evaporation is impaired in children, as they have lower sweat rate and electrolyte concentration in the heat than adults. Pre-exercise assessment of hydration status is important to avoid children starting to exercise in a dehydrated state. As they often do not drink enough to avoid dehydration while exercising in the heat, they should be educated to drink before, during and after exercise, especially in the heat.

KEYWORDS: Thermoregulation; dehydration; sweat; electrolytes.

\section{Evaluación del estado hidroelectrolítico de niños practicantes de ejercicio físico y recomendación de hidratación}

RESUMEN: Este estudio aborda las características termorreguladoras y proporciona recursos de prevención y diagnóstico de déficit hidroelectrolítico y de los daños causados por el ejercicio en el calor en niños. La práctica de ejercicio en el calor puede llevar a un aumento de la temperatura central, comprometiendo los resultados y la salud de los niños. El sistema termorregulador de ellos está en desarrollo, y la eliminación del calor, por evaporación del sudor, resulta comprometida, dado que los niños presentan menor tasa de sudoresis y concentración de electrólitos en el sudor que los adultos. La evaluación del estado de hidratación antes del ejercicio puede ser importante para prevenir que el niño comience el ejercicio deshidratado y, como muchas veces no beben la cantidad de líquido necesaria para evitar la deshidratación durante el ejercicio, se les debe enseñar a hidratarse antes, durante y después del ejercicio en el calor.

PALABRAS CLAVE: Termorregulación; deshidratación; sudor; electrólitos.

\section{REFERÊNCIAS}

AMERICAN ACADEMY OF PEDIATRICS. Committee on Sports Medicine and Fitness. Promotion of healthy weight-control practices in young athletes. Pediatrics, Elk Grove Village, v. II6, n. 4, p. 1557-64, dec. 2005.

AMERICAN COLLEGE OF SPORTS MEDICINE: SAWKA, M. N. et al. American College of Sports Medicine position stand. Exercise and fluid replacement. Medicine and Science in Sports and Exercise, Madison, v. 39, p. 377-90, feb. 2007. 
AMERICAN DIETETIC ASSOCIATION, DIETITIANS OF CANADA, AND AMERICAN COLLEGE OF SPORTS MEDICINE. Nutrition and Athletic Performance. Medicine and Science in Sports and Exercise, Madison, v. 4I, n. 3, p. 709-31, mar. 2009.

ARMSTRONG, L. E. Hydration Assessment Techniques. Nutrition Reviews, Baltimore, v. 63, p. S40-54, jun. 2005.

ARMSTRONG, L. E. et al. Urinary indices during dehydration, exercise, and rehydration. International Journal of Sport Nutrition, Champaign, v. 8, n. 4, p. 345-55, dec. 1998.

ARMSTRONG, L. E. et al. Urinary indices of hydration status. International journal of Sport Nutrition, Champaign, v. 4, n. 3, p. 265-79, sept. 1994.

BAR-OR, O.; ROWLAND, T.W. "Climate, body fluids, and the exercising child". In: Pediatric Exercise Medicine: From physiologic principles to health care application. Champaign, II. United States of America: Human Kinetics, 2004, p. 69-101.

BAR-OR, O. et al. Voluntary hypohydration in 10- to 12-years-old boys. Journal of Applied Physiology, Bethesda, v. 48, p. 104-8, jan. 1980.

BERGERON, M. et al. Repeated-bout exercise in the heat in young athletes: physiological strain and perceptual responses. Journal of Applied Physiology, Bethesda, v. 106, p. 476-85, feb. 2009.

BERGERON, M.; WALLER, J. L.; MARINIK, E. L. Voluntary fluid intake and core temperature responses in adolescent tennis players: sports beverage versus water. British Journal of Sports Medicine, Loughborough, v. 40, p. 406-10, may 2006.

CASA, D. J. et al. National Athletic Trainers Association Position Statement: Fluid Replacement for Athletes. Journal of Athletic Training, Dallas, v. 35, n. 2, p. 212-24, april 2000.

DOUGHERTY, K. A. et al. Two percent dehydration impairs and six percent carbohydrate drink improves boys basketball skills. Medicine and Science in Sports and Exercise, Madison, v. 38, p. 1650-8, sept. 2006.

DRINKWATER, B. L. et al. Response of prepubertal girls and college women to work in the heat. Journal of Applied Physiology, Bethesda, v. 43, p. 1046-53, dec. 1977.

FALK, B. \& DOTAN, R. Children's thermoregulation during exercise in the heat: a revisit. Applied Physiology, Nutrition, and Metabolism, Champaign, v. 33, n. 2, p. 420-7, april 2008.

FALK, B. Effects of thermal stress during rest and exercise in the paediatric population. Sports Medicine, Auckland, v. 25, p. 221-240, april 1998.

FALK, B.; BAR-OR, O.; MACDOUGALL, J. D. Thermoregulatory responses of pre-, mid-, and late-pubertal boys to exercise in dry heat. Medicine and Science in Sports and Exercise, Madison, v. 24, p. 688-94, jun. 1992. 
FALK, B. et al. Sweat lactate in exercising children and adolescents of varying physical maturity. Journal of Applied Physiology, Bethesda, v. 7I, p. 1735-40, nov. 1991.

INBAR, O. et al. Comparison of thermoregulatory responses to exercise in dry heat among prepuberal boys, young adults and older males. Experimental Physiology, Cambridge, v. 89 , p. 67I-700, jun. 2004.

INOUE, Y.; KUWAHARA, T.; ARAKI, T.J. Maturation - and aging-related changes in heat loss effector function. Journal of Physiological Anthropology and Applied Human Science, Tokyo, v. 23, p. 289-94, nov. 2004.

IULIANO, S. et al. Examination of the self-selected fluid intake practices by junior athletes during a simulated duathlon event. International Journal of Sport Nutrition, Champaign, v. 8 , p. 10-23, marc. 1998.

KOVACS, M.S. Hydration and temperature in tennis - a practical review. Journal of Science and Medicine in Sport, Belconnen, v. 5, p. 1-9, marc. 2006.

KUTLU, M.; GULER, G. Assessment of hydration status by urinary analysis of elite junior taekwon-do athletes in preparing for competition. Journal of Sports Sciences, London, v. 24, p. 869-73, aug. 2006.

MAUGHAN, R. J. et al. Fluid and electrolyte intake and loss in elite soccer players during training. International Journal of Sport Nutrition and Exercise Metabolism, Champaign, v. I4, p. 333-46, jun. 2004.

MAUGHAN, R. J. et al. Fluid and electrolyte balance in elite male football (soccer) players training in a cool environment. Journal of Sports Sciences, London, v. 23, p. 73-9, feb. 2005.

MEYER, F. et al. Effect of age and gender on sweat lactate and ammonia concentrations during exercise in the heat. Brazilian Journal of Medical and Biological Research, São Paulo, v. 40, p. 135-43, jan. 2007.

MEYER, F; BAR-OR, O.; WILK, B. Children's perceptual responses to ingesting drinks of different composition during and following exercise in the heat. International Journal of Sport Nutrition, Champaign, v. 5, p. 13-24, marc. 1995.

MEYER, F. \& BAR-OR, O. Fluid and electrolyte loss during exercise: the pediatric angle. Sports Medicine, Auckland, v. 18, p. 4-9, marc./april 1994.

MEYER, F. Water \& electrolyte losses and replenishment in children during prolonged exercise in the heat: physiological and perceptual considerations. 1993. I7I f. Tese. (Doutorado em Fisiologia e Farmacologia). Doctor of Philosophy in Physiology and Pharmacology. McMaster University, Hamilton, Ontário - Canadá. 1993.

MEYER, F. et al. Sweat electrolyte loss during exercise in the heat: effects of gender and maturation. Medicine and Science in Sports and Exercise, Madison, v. 24, p. 776-81, jul. 1992. 
NICHOLS, P. E. et al. Knowledge, attitudes, and behaviors regarding hydration and fluid replacement of collegiate athletes. International Journal of Sport Nutrition and Exercise Metabolism, Champaign, v. 15, p. 515-527, oct. 2005.

OPPLINGER, R. A. et al. Accuracy of urine specific gravity and osmolality as indicators of hydration status. International Journal of Sport Nutrition and Exercise Metabolism, Champaign, v. I5, n. 3, p. 236-5, jun. 2005.

PALACIOS, C.; WIGERTZ, K.; WEAVER, C. M. Comparison of 24-hour whole body versus patch test for estimating body surface electrolyte losses. International Journal of Sport Nutrition and Exercise Metabolism, Champaign, v. 13, p. 479-88, dec. 2003.

PATTERSON, M. J.; GALLOWAY, S. D. R.; NIMMO, M.A. Variations in regional sweat composition in normal human males. Experimental Physiology, Cambridge, v. 85, p. 869-75, nov. 2000.

PETRIE, H. J.; STOVER, E. A.; HORSWILL, C. A. Nutritional concerns for the child and adolescent competitor. Nutrition, Burbank, v. 20, p. 620-31, jul./aug. 2004.

POPOWSKI, L. A. et al. Blood and urinary measures of hydration status during progressive acute dehydration. Medicine and Science in Sports and Exercise, Madison, v. 33, n. 5, p. 74753 , may 2001 .

QUINTON, P. M.; TORMEY, J. M. Localization of Na/K-APTase in the secretory and reabsortive epithelia of perfused eccrine sweat glands: A question to the role of the enzyme in secretion. The Journal of Membrane Biology, New York, v. 29, p. 383-99, nov./dec. 1976.

RIVERA-BROWN, A. M. et al. Voluntary drinking and hydration in trained, heat-acclimatized girls exercising in a hot and humid climate. European Journal of Applied Physiology, Berlin, v. 103, p. 109-16, feb. 2008.

RIVERA-BROWN, A. M. et al. Exercise tolerance in a hot and humid climate in heat-acclimatized girls and women. International Journal of Sports Medicine, Stuttgart - New York, v. 27, p. 94350 , may 2006.

RIVERA-BROWN, A. M. et al. Drink composition, voluntary drinking, and fluid balance in exercising trained, heat-acclimatized boys. Journal of Applied Physiology, Bethesda, v. 86, p. 78-84, feb. 1999.

RODRÍGUEZ SANTANA, J. R. et al. Effect of drink pattern and solar radiation on thermoregulation and fluid balance during exercise in chronically heat acclimatized children. American Journal of Human Biology, New York, v. 7, p. 643-50, sep./oct. 1995.

ROWLAND, T. Thermoregulation during exercise in the heat in children: old concepts revisited. Journal of Applied Physiology, Bethesda, v. 105, p. 718-724, dec. 2008. 
SAWKA, M. N. Physiological consequences of hypohydration: exercise performance and thermoregulation. Medicine and Science in Sports and Exercise, Madison, v. 24, n. 6, p. 65770, jun. 1992.

SHIRREFFS, S. M. et al. The sweating response of elite professional soccer players to training in the heat. International Journal of Sports Medicine, Stuttgart, v. 26, p. 90-5, marc. 2005.

SHIRREFFS, S. M. Markers of hydration status. European Journal of Clinical Nutrition, London, v. 57, n. 2, p. S6-9, dec. 2003.

SHIRREFFS, S. M. Restoration of fluid and electrolyte balance after exercise. Canadian Journal of Applied Physiology, Champaign, v. 26, p. S228-35, dec. 2001.

STOVER, E. A. et al. Consistently high urine specific gravity in adolescent American football players and the impact of an acute drinking strategy. International Journal of Sports Medicine, Stuttgart, v. 27, p. 330-5, may 2006.

VERDE, T. et al. Sweat composition in exercise in the heat. Journal of Applied Physiology, Bethesda, v. 52, p. 1540-5, jun. 1982.

WILK, B.; RIVERA-BROWN, A.M.; BAR-OR, O. Voluntary drinking and hydration in nonacclimatized girls exercising in the heat. European Journal of Applied Physiology, Berlin, v. I0 I, n. 6, p. 727-34, nov./dec. 2007.

WILK, B.; YOXIU, H.; BAR-OR, O. Effect of body hypohydration on aerobic performance of boys who exercise in the heat. Medicine and Science in Sports and Exercise, Madison, v. 34, n. 5, p. S48, may 2002.

WILK, B.; BAR-Or, O. Effect of drink flavor and $\mathrm{NaCl}$ on voluntary drinking and hydration in boys exercising in the heat. Journal of Applied Physiology, Bethesda, v. 80, p. I I I2-7, apr. 1996.

Recebido: 15 jun. 2010

Aprovado: 04 jan. 2011

Endereço para correspondência:

Dra. Claudia Altmayer Perrone Rua Pintassilgo I 85 ap. I 63 bl I - Bairro Moema

São Paulo, SP, Brasil

CEP 045 | 4-030 